\title{
Attributional and relational processing in pigeons
}

\author{
Dennis Garlick ${ }^{1}$, Dana J. Gant ${ }^{1}$, Linda A. W. Brakel' ${ }^{2}$ and Aaron P. Blaisdell ${ }^{1 *}$ \\ Department of Psychology, University of California, Los Angeles, CA, USA \\ 2 Department of Psychiatry, University of Michigan, Ann Arbor, MI, USA
}

\author{
Edited by: \\ Patrizia D'ettorre, University of Paris \\ 13, France \\ Reviewed by: \\ William Thomas Swaney, Utrecht \\ University, Netherlands \\ Patrizia D'ettorre, University of Paris \\ 13, France \\ Francesco Bonadonna, CNRS, France \\ *Correspondence: \\ Aaron P. Blaisdell, Department of \\ Psychology, University of California Los \\ Angeles, 1285 Franz Hall, Los Angeles, \\ CA 90095-1563, USA. \\ e-mail: blaisdell@psych.ucla.edu
}

Six pigeons were trained using a matching-to-sample procedure where sample and rewarded comparisons matched on both attributional (color) and relational (horizontal or vertical orientation) dimensions. Probes then evaluated the pigeons' preference to comparisons that varied in these dimensions. A strong preference was found for the attribute of color. The discrimination was not found to transfer to novel colors, however, suggesting that a general color rule had not been learned. Further, when color could not be used to guide responding, some influence of other attributional cues such as shape, but not relational cues, was found. We conclude that pigeons based their performance on attributional properties of but not on relational properties between elements in our matching-to-sample procedure. Future studies should look at examining other attributes to compare attributional versus relational processing.

Keywords: pigeons, attributional processing, relational processing, same, different
ATTRIBUTIONAL AND RELATIONAL PROCESSING IN PIGEONS Sensitivity to similarity or difference is fundamental to successful behavior (James, 1890). Events viewed as being similar allows transfer of learning about one event to the other. Perception of events as different, on the other hand, can mitigate unwarranted generalization between them. Much animal research on same/different judgments has focused on pigeons due to their long history as visual psychophysical subjects (Cook, 2001). This research has shown that pigeons can perform well in discrimination procedures that involve matching-to-sample, oddity-from-sample, and same-different decisions (e.g., Zentall and Hogan, 1974; Young and Wasserman, 1997; Blaisdell and Cook, 2005; Cook and Wasserman, 2006; Katz et al., 2007).

Early studies utilizing the matching-to-sample procedure found only limited evidence that pigeons learned to use a rule-based, conceptual strategy that could generalize to novel stimuli (Cumming and Berryman, 1961). Instead, pigeons most likely learned specific sample-comparison pairs and nothing more (but see Zentall et al., 1984, for some evidence for abstract concept learning in matchingto-sample procedures in pigeons). More recent evidence from procedures using oddity-detection tasks (e.g., Cook et al., 1995, 1997), multi-item Same/Different discriminations (e.g., Wasserman and Young, 2010), and two-item Same/Different discrimination procedures (e.g., Blaisdell and Cook, 2005; Cook and Blaisdell, 2006; Wright, 2010) revealed moderate to strong transfer to novel test stimuli, suggesting that pigeons had used abstract relations among stimuli to solve these discriminations. The success of these more recent studies likely stems from training on larger sets of exemplars (see Wright, 2010).

Despite the large literature on relational learning in nonhuman animals, comparatively little attention has been given to the role that the dimension of similarity/dissimilarity plays in matching-to-sample or same-different discriminations (see Blough, 2001, for a review of similarity perception in pigeons). Similarity judgments can be based on matching attributes shared in common between two stimuli being compared. For example, a comparison stimulus may be judged to be similar to a sample because both stimuli are red or square, or taller than they are wide. Alternatively, a sample and comparison can be judged relationally similar when they share relations among attributes, such as both consisting of elements of the same shape or color, or in the same spatial relationship to each other. For example, a sample consisting of three black circles may be judged to be more similar to a comparison consisting of three gray squares than to a comparison consisting of two black circles and one gray square, despite the latter comparison sharing more attributes with the sample. Medin et al. (1990) reported that undergraduate student participants tended to base similarity judgments more on relational matching than attributional matching between a sample and comparisons (e.g., Figure 1).

Control by relations when humans make similarity judgments, however, appears to emerge later during development than does control by attributes. In a study of nearly 600 participants ranging in age from 3 to 80 years, Brakel et al. (2002) found young children predominantly judge relations based on attributional similarity, while preference for matching based on relational similarity did not develop fully until after the age of 7 years. Brakel et al. speculated that matching based on attributional similarity in young children may reflect control by a phylogenetically older system that is also developmentally prior to the system that governs relational control.

There is precedence in the literature on spatial behavior for the transition during childhood from control by absolute properties to control by relational properties of spatial cues. MacDonald et al. (2004) trained children ages 5-9 or adults (university undergraduate students) on a spatial-search task in which a goal item was hidden in the middle of an array of four identical landmarks. After subjects had learned to successfully find the item hidden in the center of the landmark array, expansion tests were given in which the landmarks were moved further apart yet still positioned in a 


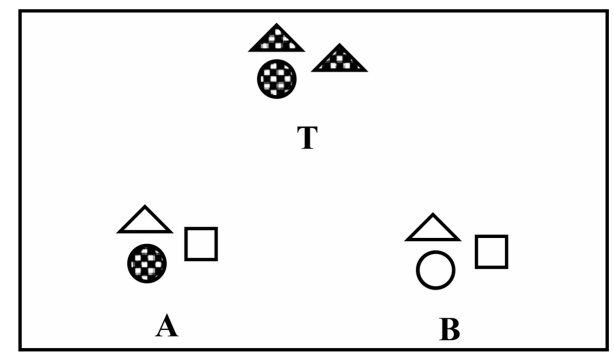

FIGURE 1 | Sample stimulus T is attributionally similar to $A$ because they both have a checkered circle. $B$ does not contain this attributional similarity to T; instead, B has a matching relation, "same-shading," with T. From Medin et al. (1990). Reprinted with permission from Psychological Science.

$4 \times 4$-array. Although adults almost invariably continued to search in the center of the expanded array, thereby evidencing control by the "middle" spatial relation, children concentrated their searches near the landmarks at approximately the absolute distance and direction from each of the landmarks at which the goal had been hidden during training. Children, therefore, were clearly controlled by the absolute properties of the individual landmarks (distance and direction) rather than by the overall relations between the landmarks and the goal. MacDonald et al. also tested common marmosets which were found to use, as did the children, absolute spatial properties rather than relational ones. Other vertebrates that have been tested also show control by absolute rather than relational spatial properties, such as gerbils (Collett et al., 1986), squirrel monkeys (Sutton et al., 2000), and pigeons (Spetch et al., 1996, 1997). This comparative evidence suggests that control by absolute spatial properties is the common ancestral condition. This system is also present in young children, with the presumably more derived relational system (Penn and Povinelli, 2007; Penn et al., 2008) emerging later in development and persisting into adulthood.

The current experiment was an effort to uncover whether pigeons, like human children below the age of 7 years, show preponderance for control by attributional rather than relational information in making a similarity judgment. Because similarity judgments based on attributional information have been shown to occur more quickly (Nickerson, 1972) and develop earlier (Brakel et al., 2002) in humans, this suggests that they might based on simpler and potentially phylogenetically older mechanisms.

\section{EXPERIMENT 1}

Pigeons were trained on the procedure shown in Figure 2A. Each trial began with the presentation of a sample stimulus consisting of a circle divided into two equal halves. Each half was a different color, such as red/green. All 12 combinations of the colors red, green, blue, and yellow were used. On half the trials the circle halves were arranged horizontally (left/right) of each other, and on the remaining trials they were in a vertical (top/bottom) arrangement. After pecking a number of times at the circle, two pairs of bars were presented below the sample, one on either side of the screen. These served as the comparison stimuli. One comparison was the same combination of colors as the sample and was placed in the same arrangement (right/left or top/bottom) as was the sample.

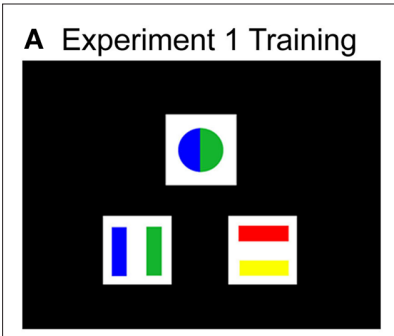

C Experiment 2 Probes

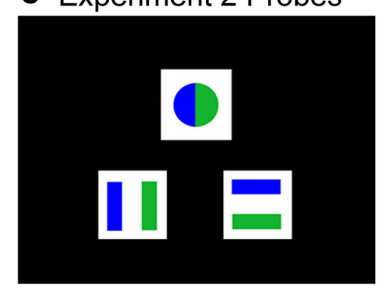

E Experiment 4 Probes

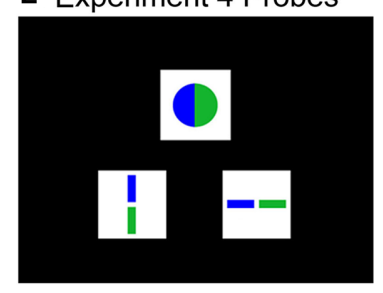

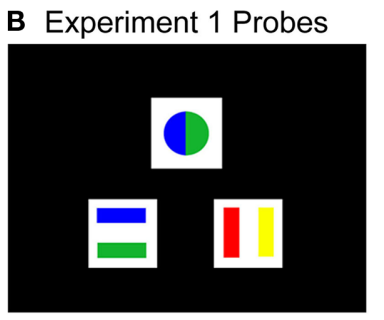

D Experiment 3 Probes

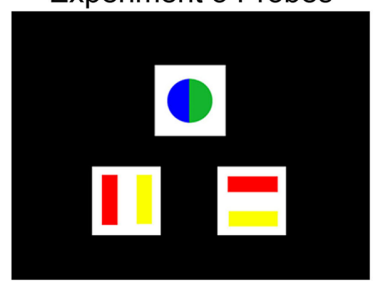

F Experiment 5 Probes

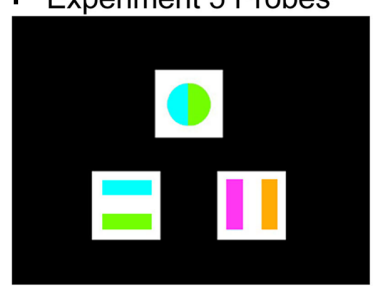

FIGURE 2 | Examples of stimulus displays presented to the pigeons. (A) An example of a training display used in Experiment 1. The circular stimulus at the top of the panel served as a sample; the pair of bars at the bottom of (A) show the rewarded comparison stimulus on the left (an attributional and relational match to the sample) and non-rewarded comparison on the right (non-matching to the sample in both attributional and relational levels). (B) An example of a test display from Experiment 1. The comparison on the left was an attributional match to the sample, the comparison on the right was a relational match to the sample. (C) Example of a test display from Experiment 2. Both comparisons were an attributional match to the sample, but only the left-hand comparison was a relational match to the sample. (D) Example of a test display from Experiment 3. Neither comparison was an attributional match to the sample; but only the left-hand comparison was a relational match to the sample. (E) Example of a test display from Experiment 4. Both comparisons were an attributional match to the sample, but only the right-hand comparison was a relational match to the sample. The orientations of the bars in the left-hand comparison were oriented in the same overall shape as the colored halves of the sample stimulus. (F) Example of a test display from Experiment 5. The comparison on the left was an attributional match, while the comparison on the right was a relational match on probe tests involving novel color elements.

The other comparison consisted of bars that were different color and arrangement than the sample. Pecks to the matching comparison were reinforced, while pecks to the non-matching comparison terminated the trial without reinforcement.

After mastering the task, non-reinforced probes were presented. On probe trials the same samples as used during training were presented, but were followed by comparisons that matched either the color (attributional match) or arrangement (relational match) of the sample elements, but not both. Thus, as illustrated in Figure 2B, if the sample was blue/green horizontal, the comparisons were blue/green vertical (attributional match) and red/yellow horizontal 
(relational match). Evidence for attributional processing would come from a bias to select the color-matched comparison, while evidence for relational processing would come from a bias toward selecting the relational-matched comparison.

\section{METHODS}

\section{Subjects}

Six experimentally naïve adult White Carneaux pigeons (Columba livia) participated in the experiment. Pigeons were individually housed in steel home-cages with metal-wire mesh floors in a vivarium, and a 12-h light-dark cycle was maintained. Testing was conducted 5-7 days a week during the light cycle. The pigeons were maintained at approximately $85 \%$ of their free-feeding weights, and were given free access to grit and water while in their home-cages. All research reported in this paper was conducted following the ethical guidelines of and approved by the University of California at Los Angeles Animal Care and Use Committee.

\section{Apparatus}

Testing was conducted in a flat-black Plexiglas chamber $(38 \mathrm{~cm}$ wide $\times 36 \mathrm{~cm}$ deep $\times 38 \mathrm{~cm}$ high). All stimuli were presented by computer on a color LCD monitor (NEC MultiSync LCD1550M) visible through a $23.2-\mathrm{cm} \times 30.5-\mathrm{cm}$ viewing window in the middle of the front panel of the chamber. Pecks to the monitor were detected by an infrared touch screen (Carroll Touch, Elotouch Systems, Fremont, CA, USA) mounted on the front panel. A $28-\mathrm{V}$ house-light located in the ceiling of the box was used for illumination, except during time outs. A food hopper (Coulbourn Instruments, Allentown, PA, USA) was located below the monitor with an access hole situated flush with the floor. All experimental events were controlled and data recorded with a Pentium III-class computer (Dell, Austin, TX, USA). A video card controlled the monitor using the SVGA graphics mode $(800 \times 600$ pixels $)$.

The same subjects apparatus used in Experiment 1 were used also for the other experiments.

\section{PROCEDURE}

\section{Preliminary training}

The six pigeons were first trained to eat from the hopper. Next, responses were autoshaped to a white disk that would appear in the center of the screen. A single peck to the disk resulted in raising the hopper for $3 \mathrm{~s}$ before lowering again. This was followed by a 60-s intertrial interval (ITI) before the next disk was displayed. Once the pigeon was consistently responding to the disk, the matching training began.

\section{Matching training}

Stimuli were presented using a simultaneous match to sample paradigm. The sample stimulus was a $140 \times 140$-pixel square that contained a circle with two different colored halves in either horizontal or vertical arrangement, as shown at the top of Figure 2A. Initially, the pigeons were rewarded for a single peck to the sample stimulus alone. Following consistent responding, the reward criterion was raised to 2, 5, and finally 10 pecks.

Once the pigeons were consistently pecking 10 times to the sample stimulus, the comparison stimuli were introduced. The comparison stimuli were two $140 \times 140$ pixel squares, each com- posed of two different colored rectangular bars in either horizontal or vertical arrangement, as shown in the bottom portion of Figure 2A. The animals were rewarded for pecking the comparison that matched in terms of both attribute (color) and relation (horizontal versus vertical). The other comparison did not match in either attribute or relation. For instance, if the sample was blue/ green in a horizontal relation, then the correct comparison would have been blue/green in the horizontal relation, and the incorrect comparison would have been red/yellow in a vertical relation. The training sessions alternated between using a white background for the stimuli (as shown in Figure 2A) and keeping the background the same black color as the rest of the screen. Since asymptotic mean accuracy was virtually identical on the white and black backgrounds, only the white background was used during testing and in subsequent experiments.

Stimulus displays consisted of combinations of four colors (red, green, blue, and yellow), two relations (horizontal and vertical), and location of correct comparison stimulus (left or right) resulting in a total of 96 possible combinations. All combinations were presented in each session in a randomized order. A session was terminated after all combinations had been presented or after $60 \mathrm{~min}$, whichever came first. Training was continued until each pigeon reached a criterion of $80 \%$ choice accuracy for two consecutive sessions.

\section{Testing}

Birds were presented with probes that compared control of choice by attributional features versus relational features (Figure 2B). For example, if the sample was blue/green horizontal, the comparisons were blue/green vertical (same attribute, different relation) and red/ yellow horizontal (same relation, different attribute). For the test sessions, test trials were intermixed with the previously presented training trials. No test trials were administered before trial 20 of the training trials, and there were at least three training trials between successive test trials. The pigeons were not rewarded during the test trials, irrespective of their choice.

\section{RESULTS}

Figure 3 illustrates acquisition across training. Two of the pigeons, George and Jerry, showed poor responding initially, slowing their acquisition. However, all birds eventually reached the performance criterion of $80 \%$ correct on two consecutive sessions by the end of acquisition. Figure 4 illustrates performance on the probes. Pigeons showed a strong preference for the comparison that matched the sample in terms of attribute rather than relation. This conclusion was supported by a dependent-sample $t$-test comparing proportion of choices to the attribute match comparison to a chance level of performance $=0.5, t(5)=20.15, p<0.001$.

\section{EXPERIMENT 2}

The finding that the attribute of color seemed to overpower control by relations led us to ask whether the birds would show control by relations when the color attribute was held constant by using the same colors as in the sample for both comparisons. To assess this, the birds were presented with comparison stimuli that possessed the same color attributes as the sample stimulus, but only one of the comparisons also matched in terms of relation (Figure 2C). 


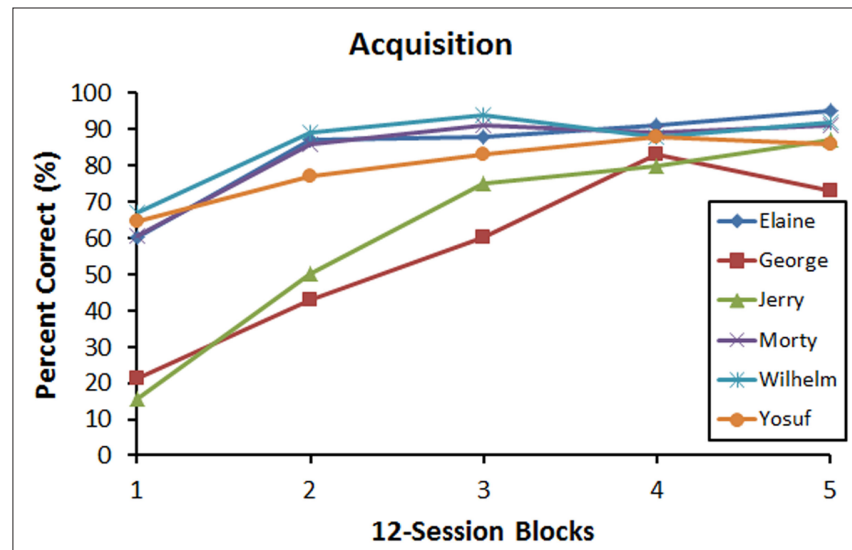

FIGURE 3 | Acquisition curves showing mean discrimination accuracy during training as a function of 12-session block. Each line depicts an individual bird.

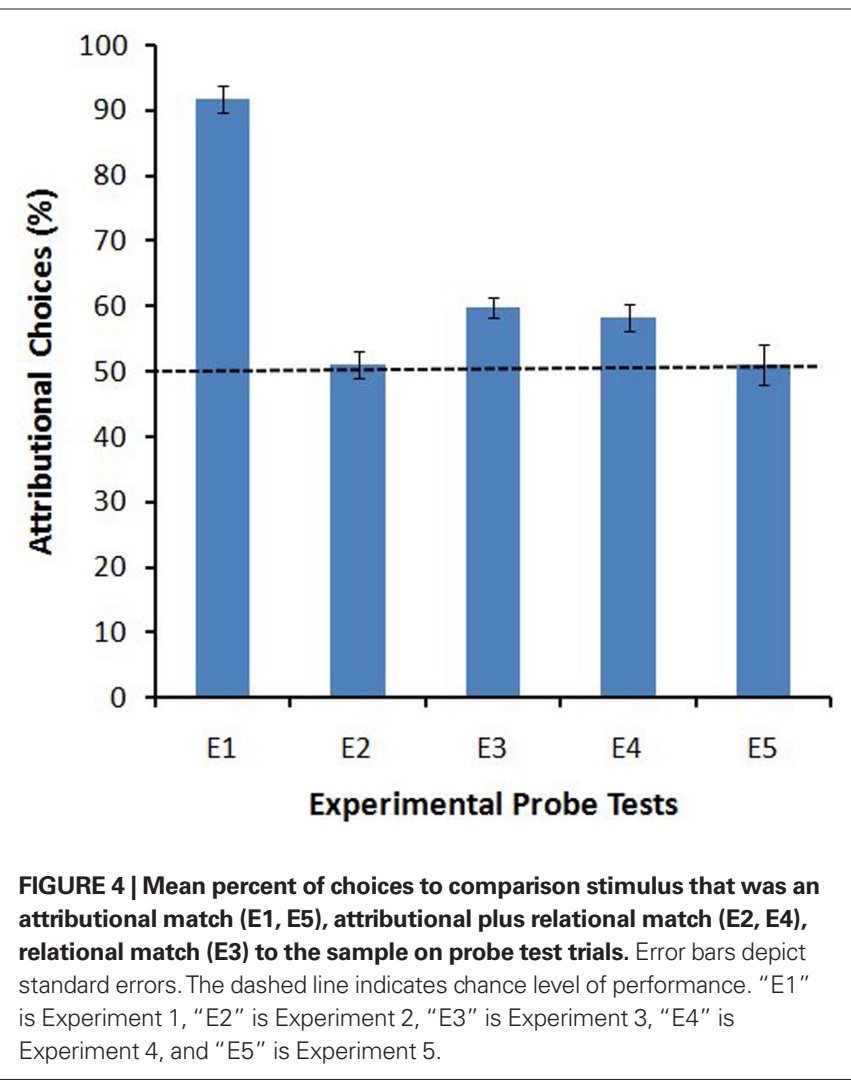

For instance, if the sample was blue/green horizontal, the comparisons were blue/green horizontal (same relations) and blue/green vertical (different relations).

\section{PROCEDURE}

\section{Training and testing}

Training was continued as in Experiment 1 for at least five sessions before testing began. Details of the testing procedure were the same as for Experiment 1 except for differences in the probe items as described above.

\section{RESULTS}

Figure 4 illustrates performance on the probes. There was no significant difference in pigeons' choices of comparison that matched attribute and relation versus attribute alone, single-sample $t$-test comparing proportion of choices to the attribute and relation match to a chance level of $0.5, t(5)=0.50, p>0.5$. This suggests that the birds did not show any sensitivity to the relations between elements, even when color attribute did not suggest an alternative response.

\section{EXPERIMENT 3}

Why was there no effect of relations in Experiment 2? Perhaps use of the same color in sample and comparisons rendered the comparisons too similar to the sample or distracted the birds from noticing the relations. In Experiment 3, we presented the birds with probes in which the comparison colors differed from the sample color. The comparisons therefore either matched or mismatched with the sample on the relation (horizontal or vertical) between elements (Figure 2D). If the difference in color between sample and comparisons makes the birds more sensitive to the relational attributes of the stimuli, then we may see control by relations.

\section{PROCEDURE}

\section{Training and testing}

Training was continued as in Experiment 1 for at least five sessions before testing began. Details of the testing procedure were the same as for Experiment 1 except for differences in the probe items as described above.

\section{RESULTS}

Morty failed to complete his probe trials and thus was not included in the analysis. Figure 4 illustrates performance on the probes. These probes revealed a significant preference for the relation that was consistent with the sample stimulus as assessed with a dependent-sample $t$-test comparing proportion of choices to the relational match against a chance level of $0.5, t(4)=6.49, p<0.01$.

\section{EXPERIMENT 4}

Our choice of stimuli included potentially confounding attributional features that may have influenced test performance. The overall shape of the elements of the sample stimulus was more similar to the shape of the elements in the matching than in the non-matching stimulus. That is, the elements in a left/right arrangement were taller than they were wide, whereas elements in a top/bottom arrangement were wider than they were tall. Also, elements in the left/right arrangement had long vertical lines, while the elements in the top/ bottom arrangement had long horizontal lines. The similarities between the sample and comparison stimuli on these dimensions (which we collectively call "shape") may have contributed to performance during training and in particular during the critical probe tests. A further test that pitted Attribute and Shape versus Attribute and Relation was used to examine control by this variable (Figure 2E). For instance, if the sample was blue/green horizontal, one of the comparisons was blue/green vertical but with the elements arranged in the same overall shape (longer height than width) as the blue/ green horizontal sample stimulus, while the other blue/green comparison was arranged horizontally (the same as the sample) but with 
shapes that did not match the blue/green vertical sample stimulus (greater width than height). To the extent that the relation between elements contributes to performance, pigeons should choose the relational matching comparison. On the other hand, to the extent that overall element shape contributes to performance, pigeons should choose the relational non-matching comparison.

\section{PROCEDURE}

\section{Training and testing}

Training was continued as in Experiment 1 for at least five sessions before testing began. Details of the testing procedure were the same as for Experiment 1 except for differences in the probe items as described above.

\section{RESULTS}

Figure 4 illustrates performance on the probes. We found a significant preference for the relational non-matching comparison as assessed by a dependent-sample $t$-test comparing proportion of choices to the relational non-matching comparison to a chance level of $0.5, t(5)=3.85, p<0.02$. These results suggest greater control by the attributional feature of element shape than by the relation between elements. This was surprising as there was not a significant preference in Experiment 2 for shape when it was presented together with the additional corresponding feature of relation.

\section{EXPERIMENT 5}

How general is the matching behavior in our task? Is it concept learning which is general or is performance restricted to the familiar training set of stimuli? To what degree does the trained behavior generalize to novel stimuli? The birds were presented with probes similar to those in Experiment 1, but the attributes (colors) were novel in that they had not been used in any of the prior training or testing (Figure 2F). For instance, if the sample was aqua/lime horizontal, the comparisons were aqua/lime vertical (attribute same/ relation different) and fuchsia/orange horizontal (relation same/ attribute different). All 12 combinations of the colors aqua, lime, fuchsia, and orange were used.

\section{METHODS}

\section{Procedure}

Training and testing. Training was continued as in Experiment 1 for at least five sessions before testing began. Details of the testing procedure were the same as for Experiment 1 except for differences in the probe items as described above.

\section{Results}

Figure 4 illustrates performance on the probes. The pigeons did not show a significant preference for attribute over relation and shape when the colors were novel as assessed with a single-sample $t$-test comparing proportion of choices to the attribute match compared to a chance level of $0.5, t(5)=0.28, p>0.5$, unlike Experiment 1 with familiar colors.

\section{GENERAL DISCUSSION}

The most consistent result from the various probe trials was that color played a dominant role in governing responding. This suggests that the pigeons had a strong attentional bias to color attributes during the training phase, and that color attributes potentially interfered with control by other features such as relation and shape. As are all members of the Family Columbidae (doves and pigeons), rock doves (C. livia) are visual foragers. As such, they rely on their excellent color vision (Varela et al., 1993) and visual acuity in the lower-central part of the visual field (Husband and Shimizu, 2001) to detect food items. Pigeons develop a strong ability to distinguish food from non-food items from visual properties alone (Balsam et al., 1992; Balsam and Deich, 2001) and have excellent color perception and discrimination abilities (Emmerton and Delius, 1980; see review by Husband and Shimizu, 2001). Furthermore, Cook has repeatedly found pigeons to show more sensitivity to color attributes than to shape attributes in both visual search and in same-different discrimination tasks (e.g., Cook, 1992; Blaisdell and Cook, 2005; Cook et al., 1996). The failure to generalize to novel colors in our task indicates that the birds did not learn the general rule of color matching, and only learned to match specific colors with themselves (e.g., green to green; cf. Cumming and Berryman, 1961).

In contrast, the results were mixed in terms of whether some other information was also acquired during the training phase. There was originally no preference between comparisons that were either the correct or incorrect in terms of orientation and shape, when both comparisons were of the sample color. Interestingly, there was some evidence that shape could govern responding, either when the comparison stimuli were different in presentation format than were prior comparison probes, or when the sample and comparison colors did not match. One explanation is that when the colors do match and the comparison stimuli are stimuli that have been previously rewarded, the birds will then automatically choose one of these stimuli without considering further cues. On the other hand, when neither of the comparisons were obviously previously related to reward - either because of a different presentation format or because the colors did not match those of the sample stimulus - then the bird will show a sensitivity to other attributional cues such as shape to determine their response.

While responding in the current study was dominated by attributes rather than relations, it is not possible to determine whether this dominance of attributes over relations is general, or specific to color. Given that it is likely that color is a very salient feature to pigeons, it may be that use of other attributes such as texture, shape, or size, may allow for better control by stimulus relations. In this case, the lower salience of the attribute may mean that it does not interfere with control by relational level features of the stimuli.

In summary, the study revealed that when pigeons are provided with multiple cues to make their judgments, they mostly used information about attribute, at least when the attribute is color. At the same time, the study did provide some evidence that the attribute of shape could also influence responding and, even more interestingly, that the ability of shape to influence responding might be mediated by the presence or absence of other, more salient cues during testing. Nevertheless, there was no evidence for the use of relational properties of the stimuli by the pigeons, although again the presence of the highly salient color attribute may have interfered with control by this information. Further studies using attributes other than color are likely to be necessary to address 
this hypothesis. The lack of evidence for behavioral control by relational similarity in pigeons may indicate that such control is found only in humans and perhaps in our nearest relatives. A recent study of relational similarity among different species of great ape and humans revealed different degrees of perception of relational similarity (Haun and Call, 2009). Although all species of great ape showed sensitivity to relational similarity among items when elements were connected by logico-causal relations; only bonobos, chimpanzees, and children over 4 years of age also processed relational similarity when elements were connected

\section{REFERENCES}

Balsam, P., and Deich, J. D. (2001). "Development of pecking in ring doves" in Avian Visual Cognition, ed. R. G. Cook. Available at: www.pigeon. psy.tufts.edu/avc/balsam/

Balsam, P., Graf, J. S., and Silver, R. (1992). Operant and Pavlovian contributions to the ontogeny of pecking in ring doves. Dev. Psychobiol. 25, 389-410.

Blaisdell, A. P., and Cook, R. G. (2005). Two-item same-different concept learning in pigeons. Learn. Behav. 33, 67-77.

Blough, D. S. (2001). "The perception of similarity," in Avian Visual Cognition, ed. R. G. Cook. Available at: www. pigeon.psy.tufts.edu/avc/dblough/

Brakel, L. A. W., Shevrin, H., and Villa, K. K. (2002). The priority of primary process categorizing: experimental evidence supporting a psychoanalytic developmental hypothesis. J.Am. Psychoanal. Assoc. 50, 483-505.

Collett, T. S., Cartwright, B.A., and Smith, B. A. (1986). Landmark learning and visuo-spatial memories in gerbils. $J$. Comp. Physiol. A 158, 835-851.

Cook, R. G. (1992). Dimensional organization and texture discrimination in pigeons. J. Exp. Psychol. Anim. Behav. Process. 18, 354-363.

Cook, R.G. (2001). Avian Visual Cognition. Available at: www.pigeon.psy.tufts. edu/avcl

Cook, R. G., and Blaisdell, A. P. (2006). Short-term item memory in successive same-different discriminations. Behav. Processes 72, 255-224.

Cook, R. G., Cavoto, K. K., and Cavoto, B. R. (1995). Same-different texture discrimination and concept learning by pigeons. J. Exp. Psychol. Anim. Behav. Process. 21, 253-260.

Cook, R. G., Cavoto, K. K., and Cavoto, B. R. (1996). Mechanisms of multidimensional grouping, fusion, and search in avian texture discrimination. Anim. Learn. Behav. 24, 150-167. (1997). Pigeon same-different concept learning with multiple stimulus classes. J. Exp. Psychol. Anim. Behav. Process. 23, 417-433.

Cook, R. G., and Wasserman, E.A. (2006). "Relational discrimination learning in pigeons," in Comparative Cognition, eds E. A. Wasserman and T. R. Zentall (New York, NY: Oxford University Press), 307-324.

Cumming, W. W., and Berryman, R. (1961). Some data on matching behavior in the pigeon. J. Exp. Anal. Behav. 4, 281-284.

Emmerton, J., and Delius, J. D. (1980). Wavelength discrimination in the "visible" and ultraviolet spectrum in pigeons. J. Comp. Physiol. A 141, 47-52.

Haun, D. B. M., and Call, J. (2009). Great apes' capacities to recognize relational similarity. Cognition 110, 147-159.

Husband, S., and Shimizu, T. (2001). "Evolution of the avian visual system," in Avian Visual Cognition, ed. R. G. Cook. Available at: www.pigeon.psy. tufts.edu/avc/husband/

James, W. (1890). The Principles of Psychology, Vol. 1. New York: Holt.

Katz, J. S., Wright, A. A., and Bodily, K. D. (2007). Issues in the comparative cognition of abstract-concept learning. Comp. Cogn. Behav. Rev. 2, 79-92.

MacDonald, S. E., Spetch, M. L., Kelly, D. M., and Cheng, K. (2004). Strategies in landmark use by children, adults, and marmoset monkeys. Learn. Motiv. 35, 322-347.

Medin, D., Goldstone, R., and Gentner, D. (1990). Similarity involving attributes and relations: judgments of similarity and differences are not inverses. Psychol. Sci. 1, 64-69. Cook, R. G., Katz, J. S., and Cavoto, B. R.

by non-causal relations. These data suggest that perception of relational similarity may be a synapomorphy shared only among great apes and not among non-ape primates (Thompson and Oden, 2000).

\section{ACKNOWLEDGMENTS}

Thanks go to Seth Resnick for his contributions at the early stages of this project, and to Hwee Cheei Lim for her assistance in managing the animal vivarium and lab staff. This research was supported by Grant MH066855 (Aaron P. Blaisdell).

Nickerson, R. S. (1972). Binary classification reaction time: a review of some studies of human information-processing capabilities. Psychon. Monogr. Suppl. 4, 275-317.

Penn, D., and Povinelli, D. J. (2007). Causal cognition in human and nonhuman animals: a comparative, critical review. Annu. Rev. Psychol. 58, 97-118.

Penn, D. C., Holyoak, K. J., and Povinelli, D. J. (2008). Darwin's mistake: explaining the discontinuity between human and nonhuman minds. Brain Behav. Sci. 31, 109-178.

Spetch, M. L., Cheng, K., and MacDonald, S. E. (1996). Learning the configuration of a landmark array: I, touch-screen studies with pigeons and humans. J. Comp. Psychol. 110, 55-68.

Spetch, M. L., Cheng, K., MacDonald, S. E., Linkenhoker, B. A., Kelly, D. M., and Doerkson, S. (1997). Learning the configuration of a landmark array in pigeons and humans: II, generality across search tasks. J. Comp. Psychol. 111, 14-24.

Sutton, J. E., Olthof, A., and Roberts, W. A. (2000). Landmark use by squirrel monkeys (Saimiri sciureus). Anim. Learn. Behav. 28, 28-42.

Thompson, R. K. R., and Oden, D. L. (2000). Categorical perception and conceptual judgments by nonhuman primates: the paleological monkey and the analogical ape. Cogn. Sci. 24, 363-396.

Varela, F. J., Palacios, A. G., and Goldsmith, T. H. (1993). "Color vision of birds," in Vision, Brain, and Behavior in Birds, eds H. P. Zeigler and H.-J. Bischof (Cambridge, MA: MIT Press), 77-98.

Wasserman, E.A., and Young, M.E. (2010) Same-different discriminations: the keel and backbone of thought and reasoning. J. Exp. Psychol.Anim. Behav. Process. 36, 3-22.

Wright, A.A. (2010). Functional relationships for determining similarities and differences in comparative cognition. Behav. Processes 85, 246-251.

Young, M. E., and Wasserman, E. A. (1997). Entropy detection by pigeons: response to mixed visual displays after same-different discrimination training. J. Exp. Psychol. Anim. Behav. Process. 23, 157-170.

Zentall, T.R.,Edwards, C.A., and Hogan,D. E. (1984). "Pigeons' use of identity," in Quantitative Analyses of Behavior, Vol. 4, eds M.L.Commons, R. J. Herrnstein, and A. R. Wagner (Cambridge, MA: Ballinger), 273-293.

Zentall, T. R., and Hogan, D. E. (1974) Abstract concept learning in the pigeon. J. Exp. Psychol. 102, 393-398.

Conflict of Interest Statement: The authors declare that the research was conducted in the absence of any commercial or financial relationships that could be construed as a potential conflict of interest.

Received: 04 October 2010; accepted: 16 January 2011; published online: 09 February 2011.

Citation: Garlick D, Gant DJ, Brakel LAW and Blaisdell AP (2011) Attributional and relational processing in pigeons. Front. Psychology 2:14. doi: 10.3389/ fpsyg.2011.00014

This article was submitted to Frontiers in Comparative Psychology, a specialty of Frontiers in Psychology.

Copyright (c) 2011 Garlick, Gant, Brakel and Blaisdell. This is an open-access article subject to an exclusive license agreement between the authors and Frontiers Media $S A$, which permits unrestricted use, distribution, and reproduction in any medium, provided the original authors and source are credited. 